\title{
BIBECHANA
}

A Multidisciplinary Journal of Science, Technology and Mathematics ISSN 2091-0762 (Print), 2382-5340 (0nline)

Journal homepage: http://nepjol.info/index.php/BIBECHANA

Publisher: Research Council of Science and Technology, Biratnagar, Nepal

\section{The non-linear optical crystal growth and characterization of piperizantum p- aminobentzone}

\author{
M. Vallikkodi", S. Sudhahar \\ Department of Physics, Alagappa University, Karaikudi- 630 004, India. \\ *Email: vallikkodi.10@gmail.com \\ Article history: Received 09 February, 2018; Accepted 3 August, 2018 \\ DOI: http://dx.doi.org/10.3126/bibechana.v16i0.20875 \\ This work is licensed under the Creative Commons CC BY-NC License. \\ https://creativecommons.org/licenses/by-nc/4.0/

\section{(c) (7) (8)}

\begin{abstract}
The nonlinear optical properties of piperizanium $\mathrm{p}-$ aminobenzoate (PZPAB) crystal were successfully grown with the help of temperature gradient (Slow evaporation) method. The good quality of PZPAB single crystal is formed. The PZPAB is characterized with the help of X-ray diffraction (XRD), Fourier transform infrared (FTIR) measurement, photoluminescence (PL), Raman spectroscopy, ultra violet visible spectroscopy (UV) and finally etching analysis was done.
\end{abstract}

Keywords: Piperizanium p - aminobenzoate; nonlinear optical crystal; slow evaporation method.

\section{Introduction}

A material that has good crystalline nature is also having good optical and electrical properties [1-3]. Many organic and inorganic crystal materials had high polarization ability and are good candidates for nonlinear optical (NLO) studies [4]. However, the net polarization depends on the material symmetry properties, with respect to the impinging fields. So far, there are many organic crystals which is used for NLO yet, piperizanium p- aminobenzoate having the adorable nature of nonlinear properties. In the present article, gives the advancement of new NLO material (piperizanium p- aminobenzoate); which is being characterized with the help of XRD, FTIR, UV, Raman and PL.

\section{Materials and Methods}

Piperazinium p-aminobenzoate crystal was prepared in the room temperature by slow evaporation growth method [5] by using as a methanol solvent. The precursor's materials of Piperazine (98\%) and p-aminobenzoic acid (99\%) were taken in the equimolar ratio of 1:1 for the synthesis process is shown in figure 1. The p-Aminobenzoic acid was first dissolved in the solvent of methonal, after 
complete dissolved acid material and then the base compound of Piperazine was added little by little with the acid solution. The solution is allowed to get a homogenous mixture by continuously stirring for 8 hours using the temperature control magnetic stirrer in the room temperature circumstances [5]. After attaining the homogenous saturated state the solution was filtered using the whatman filter paper which is having the fine holes in the range of $110 \mu \mathrm{m}$ and the filtered solution was covered with the perforated sheet having the fine holes for the evaporation and it was kept at the room temperature without disturbance. After 2 successive recrystallization process the good quality PZPAB crystal was harvested with in the span of 60 days. The figure 2 shows the crystal of PZPAB.

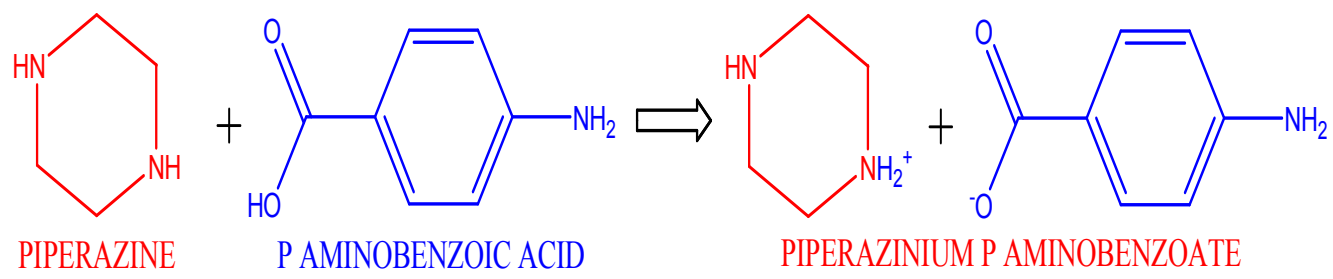

Fig. 1: Synthesis scheme of Piperazinium P-Aminobenzoate.

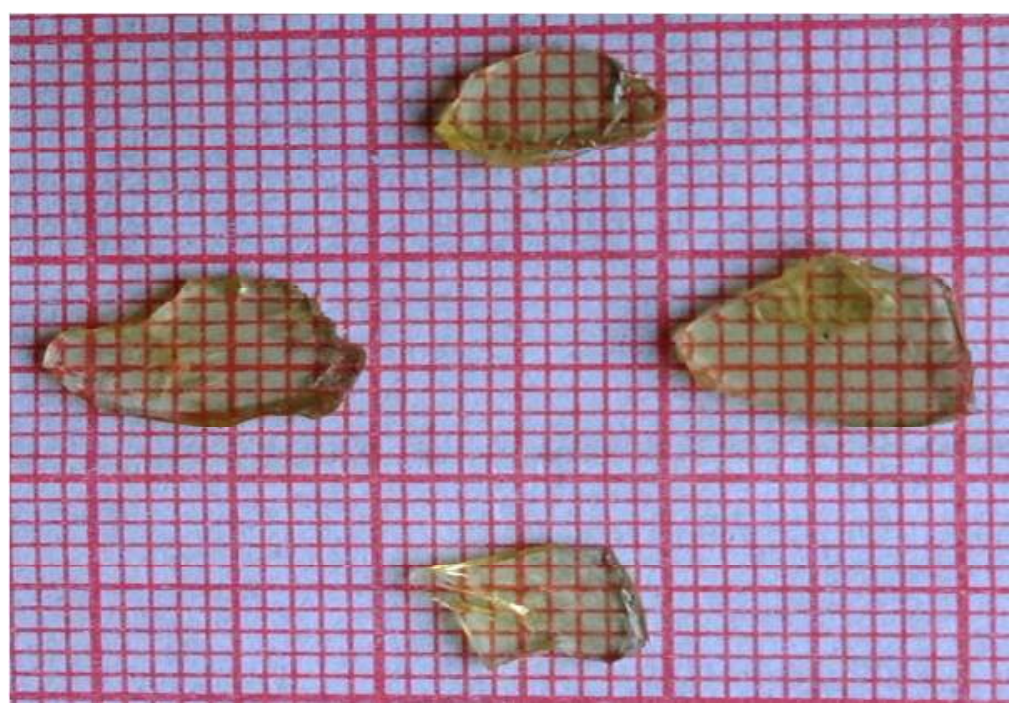

Fig. 2: Grown crystal of Piperazinium P-Aminobenzoate.

\section{Results and Discussion}

\section{XRD Analysis}

(i) The single crystal X-ray diffraction analysis is the powerful tool for determination of the structure of the crystal. The Piperazinium P-Aminobenzoate crystal belongs to orthorhombic crystal system with noncentrosymmetric space group Pna2 ${ }_{1}$. This space group satisfies one of the basic essential requirements of NLO material. The lattice parameters of the Piperazinium P-Aminobenzoate is found 
to $\mathrm{be} a=18.2964 \AA, \mathrm{b}=7.1388 \AA, \mathrm{c}=10.3574 \AA, \alpha=90^{\circ}, \beta=90^{\circ}, \gamma=90^{\circ}$ and volume $\mathrm{V}=1352.83$ $\AA^{3}$. The structural parameters of the Piperazinium P-Aminobenzoate crystal were listed in the table 1 .

Table 1: PZPAB crystal.

\begin{tabular}{|l|l|}
\hline Crystal property & Piperazinium P-Aminobenzoate \\
\hline Empirical formula & $\mathrm{C}_{4} \mathrm{H}_{11} \mathrm{~N}_{2}{ }^{+} \cdot \mathrm{C}_{7} \mathrm{H}_{6} \mathrm{NO}_{2} \cdot \mathrm{H}_{2} \mathrm{O}$ \\
\hline Crystal system & Orthorhombic \\
\hline Spacegroup & $\mathrm{Pna}_{1}$ \\
\hline Unit cell parameters & $\mathrm{a}=18.2964 \AA, \mathrm{b}=7.1388 \AA$, \\
& $\mathrm{c}=10.3574 \AA$, \\
& $\alpha=90^{\circ}, \beta=90^{\circ}, \gamma=90^{\circ}$ \\
\hline Volume of the unit cell & $\mathrm{V}=1352.83 \AA^{3}$ \\
\hline Radiation wavelength & $\lambda=0.71073 \AA$ \\
\hline
\end{tabular}

The crystalline nature of the Piperazinium p-aminobenzoic crystal was characterized by Powder x-ray diffraction analysis to reveal crystalline perfection of the compound. The x-ray diffraction spectrum of PZPAB was recorded using the X'pert PRO powder diffractometer with $\mathrm{CuK}_{\alpha}$ radiation having the wavelength of $\lambda=1.5406 \AA$. The sample was scanned from the range of $10^{\circ}$ to $80^{\circ}$ at the rate of $2 \%$ minute. The recorded powder x-ray diffraction spectrum is shown in Figure 3. The presence of sharp and well defined peaks confirms the good crystalline nature of piperazinium p-aminobenzoate crystal and the corresponding peaks were indexed. The sharp intense peaks are shown in the table 2 .

\section{FTIR Analysis}

The FTIR spectrum was recorded to understand the chemical bonding and it provides useful information regarding the molecular structure of the compound. The $\mathrm{KBr}$ pellet technique was used to analyze the sample. FTIR spectrum was taken for the powdered sample in the wavelength range 4000$400 \mathrm{~cm}^{-1}$ using 380 FTIR Spectrophotometer having the resolution of $0.5 \mathrm{~cm}^{-1}$ and the spectrum of FTIR is shown in Figure 4.The observed FTIR is summarized in table 3.

\section{Raman Analysis}

Laser Raman spectrum was taken for the powdered sample in the wavelength range $4000-400 \mathrm{~cm}^{-1}$ using STR $500 \mathrm{~mm}$ focal length laser Raman spectrometer and the Raman spectrum is shown in the figure 5. Raman bands along with their vibrational assignments are summarized in table 4 .

\section{UV-Visible optical Absorption spectral analysis}

The optical absorption spectral analysis of the grown PZPAB crystal was carried out using Perkin Elmer Lambda35 spectrometer between 250 and $800 \mathrm{~nm}$. The absorption \& transmittance spectrum of the as grown PZPAB crystal is shown in the Figure 6. The UV cut off wavelength of the crystal was found to be at $317 \mathrm{~nm}$. The absence of absorption in the visible region suggests that the crystal possess the good nonlinear optical property. 
Table 2: Peaks of XRD.

\begin{tabular}{rrrrr}
\hline Pos. [ ${ }^{\circ}$ Th.] & Height [cts] & FWHM Left [02Th.] & d-spacing $[\AA]$ & Rel. Int. [\%] \\
\hline 10.4002 & 121.43 & 0.1476 & 8.50603 & 3.53 \\
13.5267 & 439.65 & 0.1476 & 6.54617 & 12.78 \\
14.1626 & 173.47 & 0.1476 & 6.25365 & 5.04 \\
15.1903 & 641.65 & 0.1476 & 5.83283 & 18.65 \\
15.5192 & 252.21 & 0.0984 & 5.70992 & 7.33 \\
17.0567 & 1184.78 & 0.1476 & 5.19854 & 34.44 \\
19.5580 & 551.63 & 0.1476 & 4.53897 & 16.04 \\
21.0129 & 470.57 & 0.1476 & 4.22787 & 13.68 \\
21.5337 & 3439.78 & 0.1476 & 4.12679 & 100.00 \\
23.8882 & 2086.44 & 0.1476 & 3.72510 & 60.66 \\
24.8055 & 1168.61 & 0.1476 & 3.58939 & 33.97 \\
26.3194 & 821.87 & 0.1476 & 3.38626 & 23.89 \\
26.8804 & 317.96 & 0.1476 & 3.31685 & 9.24 \\
27.4870 & 740.96 & 0.1476 & 3.24502 & 21.54 \\
28.3924 & 145.42 & 0.1476 & 3.14356 & 4.23 \\
29.4507 & 256.36 & 0.1476 & 3.03297 & 7.45 \\
30.5232 & 719.77 & 0.1968 & 2.92879 & 20.92 \\
31.3467 & 721.21 & 0.1476 & 2.85371 & 20.97 \\
31.9957 & 276.41 & 0.1476 & 2.79729 & 8.04 \\
32.4648 & 201.46 & 0.1968 & 2.75793 & 5.86 \\
33.7596 & 164.56 & 0.1476 & 2.65506 & 4.78 \\
34.3585 & 209.28 & 0.2952 & 2.61014 & 6.08 \\
35.1350 & 131.82 & 0.1968 & 2.55421 & 3.83 \\
35.6070 & 134.51 & 0.1476 & 2.52143 & 3.91 \\
36.3967 & 554.72 & 0.1968 & 2.46852 & 16.13 \\
37.9391 & 177.68 & 0.1476 & 2.37163 & 5.17 \\
39.0984 & 130.54 & 0.2460 & 2.30395 & 3.80 \\
39.5630 & 76.17 & 0.1476 & 2.27795 & 2.21 \\
41.2584 & 89.98 & 0.3936 & 2.18818 & 2.62 \\
41.8669 & 81.48 & 0.1476 & 2.15778 & 2.37 \\
43.3737 & 77.32 & 0.1476 & 2.08624 & 2.25 \\
43.8176 & 84.64 & 0.1476 & 2.06613 & 2.46 \\
44.3690 & 117.18 & 0.1476 & 2.04173 & 3.41 \\
45.0060 & 82.97 & 0.1968 & 2.01430 & 2.41 \\
46.4121 & 73.75 & 0.1476 & 1.95650 & 2.14 \\
48.0597 & 46.94 & 0.1968 & 1.89320 & 1.36 \\
49.2598 & 48.17 & 0.1968 & 1.84985 & 1.40 \\
50.7274 & 282.97 & 0.2460 & 1.79973 & 8.23 \\
51.2972 & 67.33 & 0.1476 & 1.78107 & 1.96 \\
52.5555 & 50.83 & 0.2460 & 1.74135 & 1.48 \\
53.4279 & 22.86 & 0.2952 & 1.71496 & 0.66 \\
54.1358 & 19.44 & 0.2952 & 1.69420 & 0.57 \\
55.2169 & 25.97 & 0.3936 & 1.66356 & 0.76 \\
56.6958 & 36.33 & 0.2952 & 1.62363 & 1.06 \\
57.8343 & 32.12 & 0.5904 & 1.59435 & 0.93 \\
60.7458 & 28.30 & 0.5904 & 1.52472 & 0.82 \\
\hline & & & & \\
& & &
\end{tabular}


M. Vallikkodi and S. Sudhahar/BIBECHANA 16 (2019) 15-22: RCOST p.19 (Online Publication: Dec., 2018)

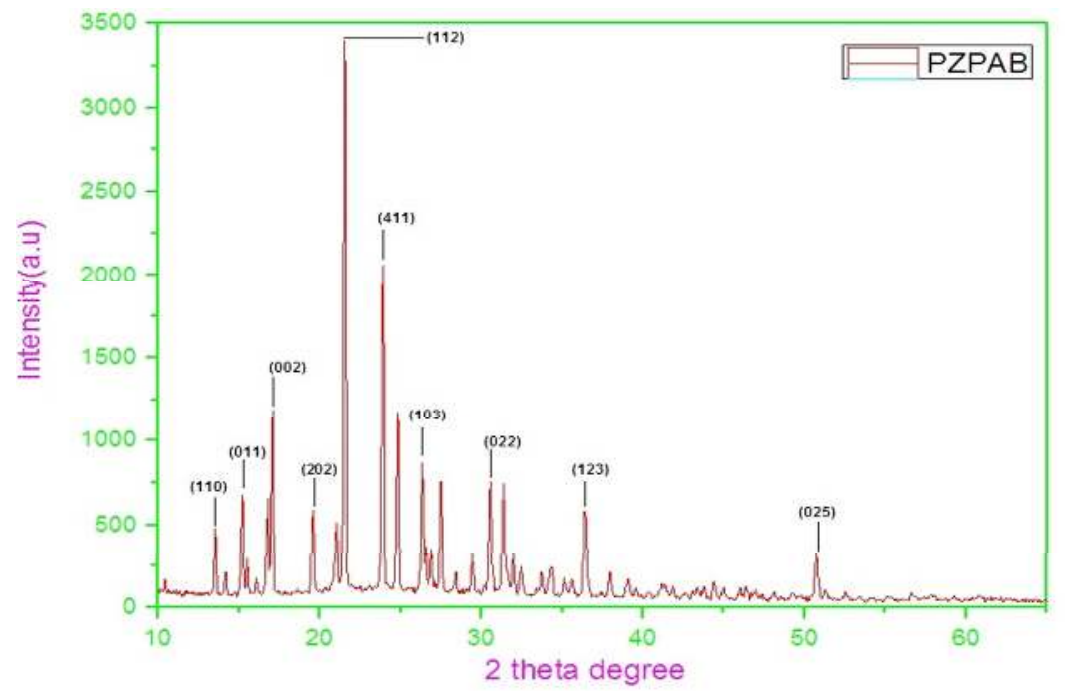

Fig. 3: Powder XRD pattern of PZPAB crystal.

Table 3: FTIR frequency assignments of PZPAB compound.

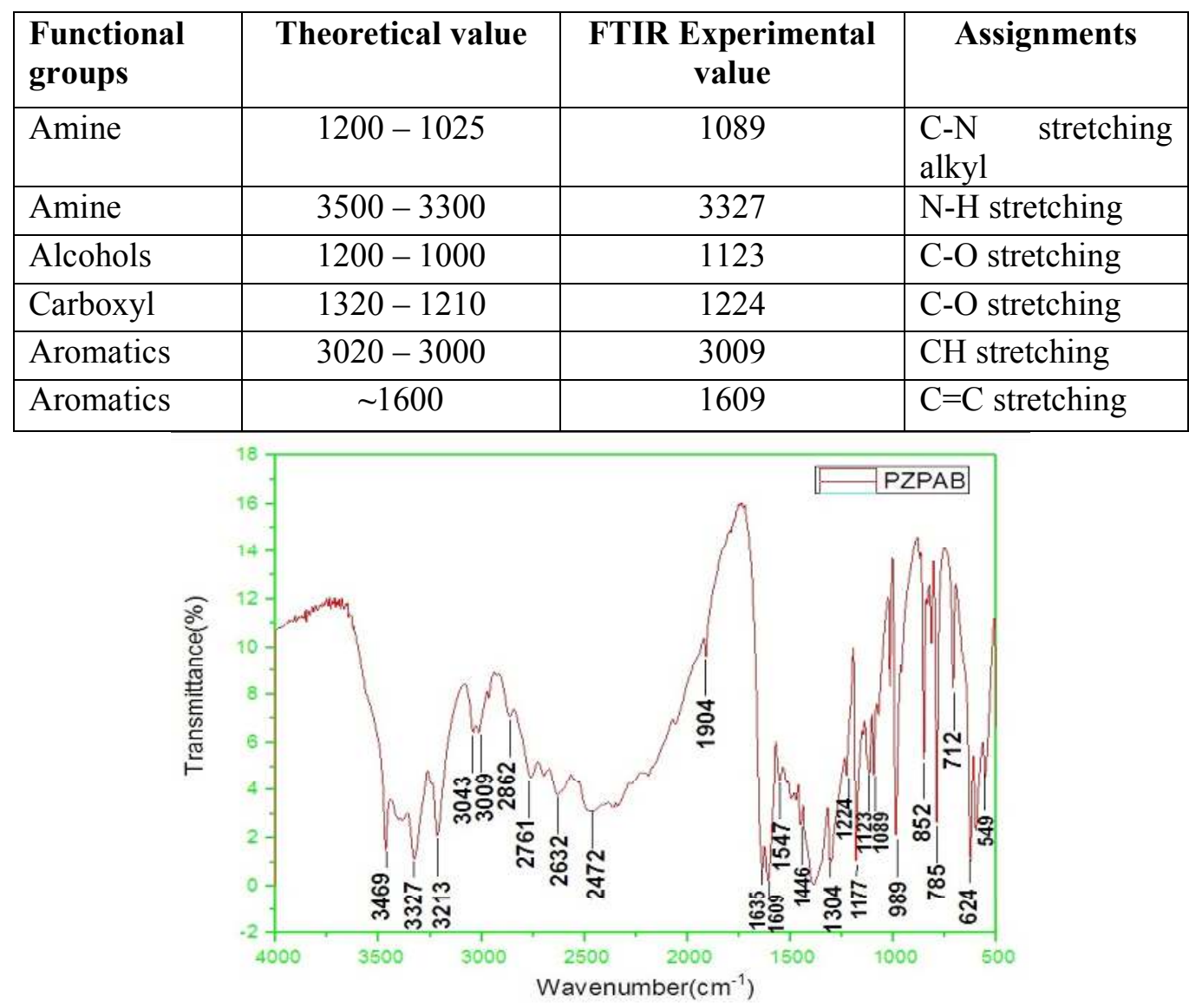

Fig. 4: FTIR spectrum of PZPAB. 
Table 4: Raman frequency assignments of PZPAB compound.

\begin{tabular}{|l|l|l|l|}
\hline \multicolumn{1}{|c|}{$\begin{array}{c}\text { Functional } \\
\text { groups }\end{array}$} & Theoretical value & \multicolumn{1}{|c|}{$\begin{array}{c}\text { Laser Raman Experimental } \\
\text { values }\end{array}$} & \multicolumn{1}{|c|}{ Assignments } \\
\hline Amine & $1200-1025$ & 1150 & C-N stretching alkyl \\
\hline Amine & $3500-3300$ & 3331 & N-H stretching \\
\hline Alcohols & $1200-1000$ & 1150 & C-O stretching \\
\hline Carboxyl & $1320-1210$ & ---- & C-O stretching \\
\hline Aromatics & $3020-3000$ & 3077 & CH stretching \\
\hline Aromatics & $\sim 1600$ & 1605 & C=C stretching \\
\hline
\end{tabular}

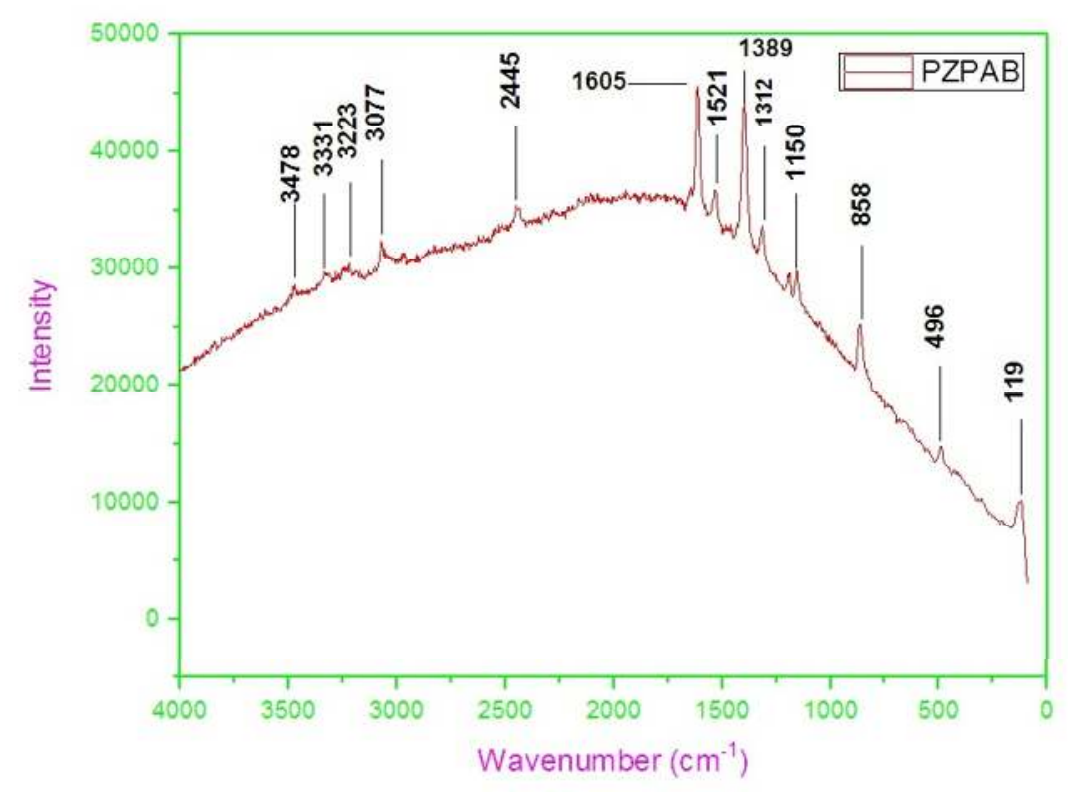

Fig. 5: Laser Raman spectrum of PZPAB.

\section{UV-Visible optical Absorption spectral analysis}

The optical absorption spectral analysis of the grown PZPAB crystal was carried out using Perkin Elmer Lambda35 spectrometer between 250 and $800 \mathrm{~nm}$. The absorption \& transmittance spectrum of the as grown PZPAB crystal is shown in the Figure 6. The UV cut off wavelength of the crystal was found to be at $317 \mathrm{~nm}$. The absence of absorption in the visible region suggests that the crystal possess the good nonlinear optical property.

\section{Photoluminescence studies}

Photo luminescence spectroscopy is a non-destructive method for finding out the electronic structure and optical behavior. The Photoluminescence spectrum of the grown PZPAB was recorded in the wavelength region between $300 \mathrm{~nm}$ and $550 \mathrm{~nm}$ using RF-5301 spectrophotometer. The PL spectrum (Figure 7) of PZPAB grown crystal is excited at $350 \mathrm{~nm}$. From the PL spectrum, one high intensity blue emission peak and one medium intensity violet emission peak was observed at $455 \mathrm{~nm}, 384 \mathrm{~nm}$ 
respectively, and it attributes to shallow defects in the band gap and more ordered structure. The sharp high intensity caused because of the similar transition occurring at the various energy levels within the band gap.
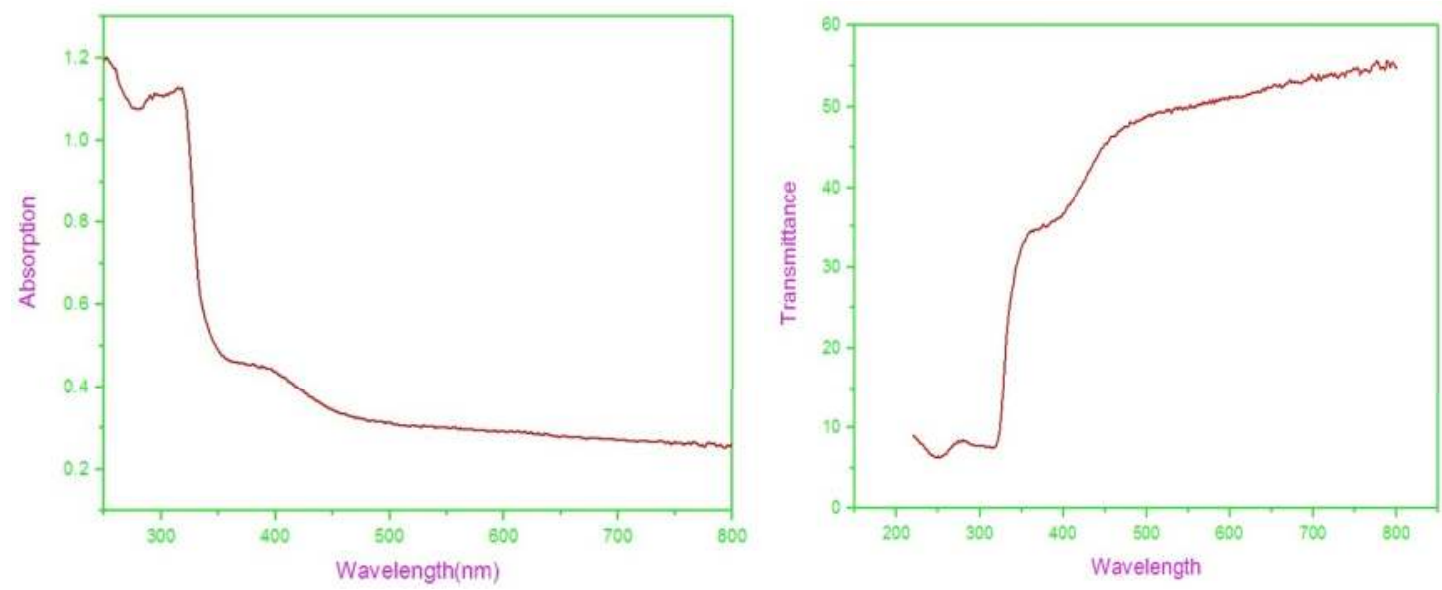

Fig. 6: UV-Visible absorption \& transmittance spectrum of PZPAB.

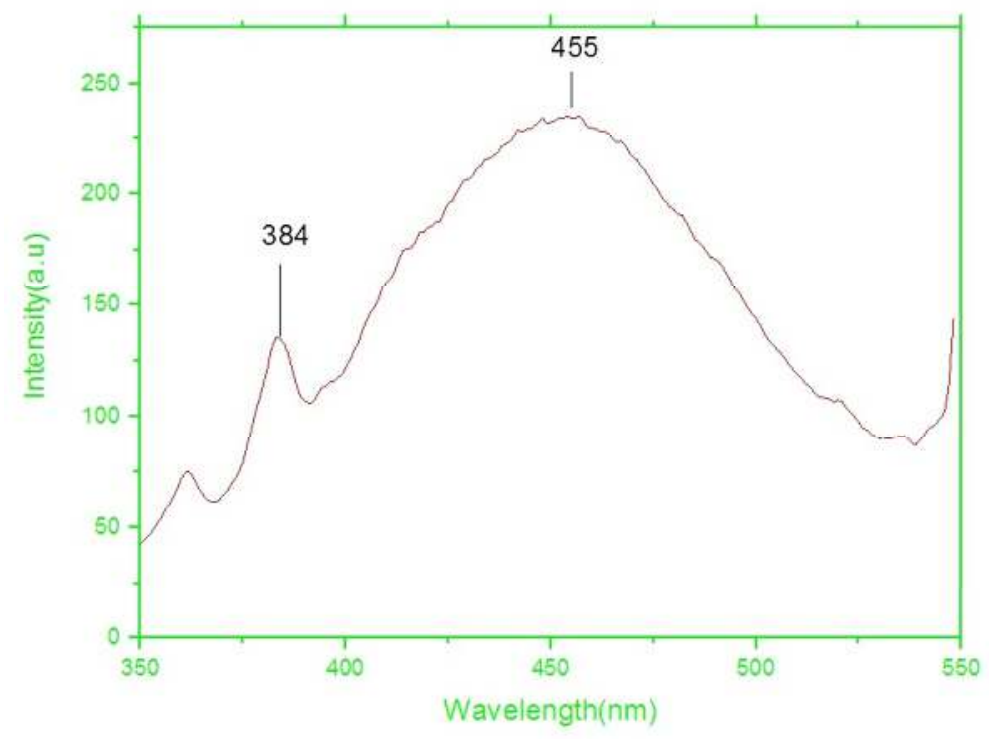

Fig. 7: Photoluminescence spectrum of PZPAB.

\section{Conclusion}

The piperazinium p-aminobenzoate crystal was successfully grown and characterized. The XRD pattern confirms the properties of NLO and the Raman and FTIR confirm all the functional groups. The UV and PL show the Optical properties. Thus, a good NLO crystal was successfully formed for LASER applications. 
M. Vallikkodi and S. Sudhahar/BIBECHANA 16 (2019) 15-22: RCOST p.22 (Online Publication: Dec., 2018)

\section{References}

[1] G. U. Sankar, A Survey on Wavelength Based Application of Ultraviolet LED Computing, 2007.

[2] C. G. Moorthy, G. U. Sankar, and G. Raj Kumar, A Design for Charging Section of Electrostatic Precipitators by Applying a Law for Electric Field Waves. Imperial Journal of Interdisciplinary Research 3(6) (2017) 842.

[3] G. U. Sankar, Climate change challenge - photosynthesis vs. hydro-electrolysis principle. Climate Change 3 (2016) 128-131.

[4] Y.R. Shen, The principles of nonlinear optics, New York, Wiley-Inter-science, 1984, p.575.

[5] H. G. Bhamidi, S. R. Wilson, R.B. Tan, P.J. Kenis, and C. F. Zukoski, Direct growth of $\gamma$-glycine from neutral aqueous solutions by slow, evaporation-driven crystallization, Crystal growth \& design 6(8) (2006) 1746-1749. 